\title{
Van der Waals-like Equations of State. I. Application to Pure Liquids
}

\author{
Koichi Fujisawa, Tomoo ShIOMI, Fumiyuki Hamada, ${ }^{*}$ \\ and Akio NAKAJIMA \\ Department of Polymer Chemistry, Faculty of Engineering, \\ Kyoto University, Kyoto 606, Japan.
}

(Received January 16, 1981)

\begin{abstract}
The applicability of six equations of state, one of which was newly proposed by us, to $n$-alkane liquids was examined with respect to the corresponding states principle. Among the equations of state for hard spheres combined with the attractive energy of the van der Waals type, our new equation (the FHN equation) reproduced fairly well the observed thermodynamic relations, and the reduction parameters calculated by this equation were least dependent on temperature. The Flory equation also gave equally good results. When the equations of state for hard spheres were combined with the Lennard-Jones $(m, n)$ potential, the Thiele equation gave better results than other equations. Furthermore, the Flory and FHN equations with the van der Waals attractive energy were compared with each other for pure polymer liquids and some small molecules except for $n$-alkanes. The FHN equation gave better agreement with the experimental values of the specific volume and the thermal expansivity than did the Flory equation. Also, the FHN equation was more satisfactory for the pressure dependence of polymer liquid volumes.

KEY WORDS Van der Waals-like Equation of State / Corresponding States Principle / Reduction Parameters / $n$-Alkane /
\end{abstract}

The Flory theory ${ }^{1,2}$ which takes the equation of state into account has been applied to various nonpolar mixtures including polymer solutions. ${ }^{3-7}$ This theory is formulated on the corresponding states principle and evaluates the reduction parameters, $p^{*}, v^{*}$ and $T^{*}$, from thermodynamic properties of the liquid such as the molar volume $V$, the thermal expansivities $\alpha$, and the thermal pressure coefficient $\gamma$. This method of evaluation of reduction parameters may be better than other methods (which use, e.g., data for the second virial coefficient of the gas and the critical constants) when the dense fluids at low temperature (far from the gas-liquid critical temperature) and specially polymer liquids are treated. However, reduction parameters, which should be independent of temperature, obtained by Flory's method vary with temperature. This leads to the conclusion that the theory is incapable of representing the $P-V-T$ relation well.

Longuet-Higgins and Widom ${ }^{8}$ obtained an equa-

\footnotetext{
* To whom correspondence should be addressed.
}

tion of state by combining the hard-sphere repulsion with the van der Waals attraction and showed that its use gave a good representation of the melting point of argon. We refer to this equation as the van der Waals-like equation of state. The Flory equation can be regarded as one of the van der Waals-like equations of state. Many kinds of van der Waals-like equations have been proposed up to the present. ${ }^{9}$ It is interesting to transform these equations to the reduced form as done by Flory and discuss their applicability to normal dense fluids.

In this paper, we deal with six van der Waals-like equations of state: the van der Waals, Guggenheim, ${ }^{10}$ Frisch, ${ }^{11}$ Thiele, ${ }^{12}$ and Flory equations, and the new equation proposed by us. First, the applicability of these equations to $n$-alkanes, which are suitable for a valid test of the corresponding states principle, is examined, and secondly, the liquids of other small molecules and polymer liquids are treated. Further, the volume dependence of the configurational energy is examined. 


\section{THEORETICAL}

If a pair potential $u(r)$ is specified by, ${ }^{13}$

$$
\begin{array}{ll}
u(r)=+\infty & (r<\sigma) \\
u(r)=\lim _{\gamma \rightarrow \infty}-\frac{\alpha}{\gamma^{3}} \exp \left(\frac{\sigma^{3}-r^{3}}{\gamma^{3}}\right) \quad(r \geqq \sigma),
\end{array}
$$

the configurational energy $E(n)$ of the system is given by,

$$
E(n)=-\left(\frac{2}{3} \pi N \alpha\right) n
$$

where $r, \sigma, N, n$, and $\alpha$ are the distance between two molecules, the diameter of the hard sphere, the number of molecules, the number density $N / V$, and a positive constant characterizing the energy of interaction for a pair of hard spheres with an attractive energy, respectively. ${ }^{13,14}$

The equation of state derived from eq 2 is,

$$
p / n k T=h(n)-a n / k T, \quad a=\frac{2}{3} \pi \alpha
$$

The function $h(n)$ is the integration constant and may be identified with the equation of state for the system of hard spheres of diameter $\sigma$. The second term $a n / k T$ is the attractive energy term. Equation 3 is called the generalized van der Waals equation of state. ${ }^{15}$ For a system of chain molecules, eq 3 may be extended to,

$$
p / n_{\mathrm{r}} k T=h\left(n_{\mathrm{r}}\right) c-a n_{\mathrm{r}} / k T
$$

where $n_{\mathrm{r}}=N r / V, r$, the number of segments, $c$, a scaling parameter related to the entropy of the system, ${ }^{16}$ and $3 \mathrm{c}$, the external degree of freedom used by Flory.

The expressions for $h(y)$ of the van der Waals, Guggenheim, ${ }^{10}$ Frisch, ${ }^{11}$ Thiele, ${ }^{12}$ and Flory ${ }^{1,2}$ equations of state are as follows:

$$
\begin{aligned}
& h(y)=(1-y)^{-1} \quad(\mathrm{vdW}) \\
& h(y)=(1-y)^{-4} \quad \text { (Guggenheim) } \\
& h(y)=\left(1+y+y^{2}\right) /(1-y)^{3} \quad \text { (Frisch) } \\
& h(y)=\left(1+2 y+3 y^{2}\right) /(1-y)^{2} \quad \text { (Thiele) } \\
& h(y)=\left(1-y^{1 / 3}\right)^{-1} \quad \text { (Flory) }
\end{aligned}
$$

where $y$ is defined by,

$$
y=(N r / V) b_{0}
$$

where $b_{0}$ is the volume of a hard sphere. The original van der Waals equation of state is represented by,

$$
h(y)=(1-4 y)^{-1}
$$

But in this study we use eq 5 , since if eq 11 is adopted, the volume of the system at $T=0$ is four times the core volume of the system.

Now let us consider the equation of state,

$$
h(y)=(1-y)^{-d}
$$

Equation 12 coincides with eq 5 for $d=1$ and with eq 6 for $d=4$. Here, we take $d$ to be 2 , and call eq 12 for this $d$ the FHN equation, i.e.,

$$
h(y)=(1-y)^{-2}
$$

If each eq 5-9 and 13 is substituted into eq 4 and transformed to the reduced form, we obtain the reduced van der Waals-like equation of state,

$$
p \tilde{v} / \tilde{T}=H(\tilde{v})-1 / \tilde{v} \tilde{T}
$$

Expressions for $H(\tilde{v})$ are given as,

$$
\begin{array}{ll}
H(\tilde{v})=\tilde{v} /(\tilde{v}-1) & (\mathrm{vdW}) \\
H(\tilde{v})=\tilde{v}^{4} /(\tilde{v}-1)^{4} & (\text { Guggenheim }) \\
H(\tilde{v})=\tilde{v}\left(\tilde{v}^{2}+\tilde{v}+1\right) /(\tilde{v}-1)^{3} & (\text { Frisch }) \\
H(\tilde{v})=\left(\tilde{v}^{2}+2 \tilde{v}+3\right) /(\tilde{v}-1)^{2} & (\text { Thiele) } \\
H(\tilde{v})=\tilde{v}^{1 / 3} /\left(\tilde{v}^{1 / 3}-1\right) & \text { (Flory) } \\
H(\tilde{v})=\tilde{v}^{2} /(\tilde{v}-1)^{2} & (\text { FHN })
\end{array}
$$

The reduced variables $\tilde{v}, \tilde{p}$ and $\tilde{T}$ are defined by,

$$
\begin{aligned}
& \tilde{v}=v / v^{*} \\
& \tilde{T}=T / T^{*} \\
& \tilde{p}=p / p^{*}
\end{aligned}
$$

where $v^{*}, T^{*}$, and $p^{*}$ are the characteristic volume per segment, temperature and pressure, respectively, and given by,

$$
\begin{aligned}
& v^{*}=b_{0} \\
& T^{*}=a / c k v^{*} \\
& p^{*}=a / v^{* 2}
\end{aligned}
$$

with $v^{*}$ the core volume of segment. In this study, we evaluate these parameters from the volume $v$, the thermal expansivity $\alpha$ and the thermal pressure coefficient $\gamma$ using the following equations at zero pressure: 


$$
\begin{aligned}
H(\tilde{v}) & =1 / \tilde{v} \tilde{T} \\
(\alpha T)^{-1} & =-\tilde{v}[\partial \ln H(\tilde{v}) / \partial \tilde{v}]_{\tilde{p}=0}-1 \\
p^{*} & =\gamma T \tilde{v}^{2}
\end{aligned}
$$

\section{VERIFICATION OF EQUATIONS OF STATE FOR $n$-ALKANES}

\section{Temperature Dependence of Thermal Expansivities}

The plot of the quantity $\left(1 / \alpha^{2}\right)(\partial \alpha / \partial T)_{p=0}$ against $\alpha T$ provides a sensitive test of the corresponding states principle. This quantity is dimensionless and needs no characteristic parameter such as $v^{*}, T^{*}$, and $p^{*} \cdot{ }^{17}$ Figure 1 shows $\left(1 / \alpha^{2}\right)(\partial \alpha / \partial T)_{p=0}$ plotted against $\alpha T$ for $n$-alkanes and polyethylene. The solid lines represent the curves observed by Flory et al. ${ }^{7}$ It is seen that the experimental curves approximately fall on a single curve, thus supporting the validity of the corresponding states principle for $n$-alkanes.

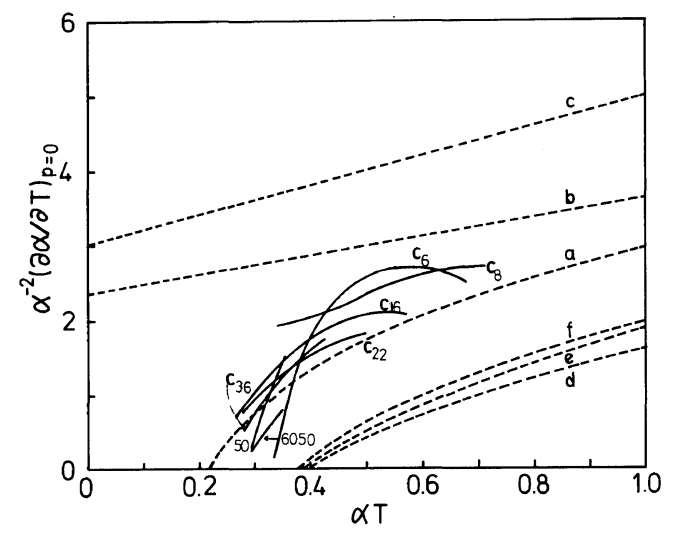

Figure 1. $\left(1 / \alpha^{2}\right)(\partial \alpha / \partial T)_{p=0}$ plotted against $\alpha T$ for $n$ alkanes. The solid lines are experimental. ${ }^{7}$ The dashed curves are theoretical: a, FHN equation; b, Flory equation; c, van der Waals equation; d, Thiele equation; e, Frische equation; f, Guggenheim equation.

The dashed curves represent the theoretical curves. From eq 28, the relation between $\left(1 / \alpha^{2}\right)(\partial \alpha / \partial T)_{p=0}$ and $\alpha T$ can be represented as,

$$
\begin{gathered}
\left(1 / \alpha^{2}\right)(\partial \alpha / \partial T)_{p=0}=\frac{\tilde{v}^{2}[\ln H(\tilde{v})]^{\prime \prime}+\tilde{v}[\ln H(\tilde{v})]^{\prime}}{-\tilde{v} \ln H(\tilde{v})-1} \\
+\tilde{v} \ln H(\tilde{v})+1
\end{gathered}
$$

Equations 28 and 30 give the following expressions for the Flory, van der Waals, Guggenheim and
FHN equations,

$$
\begin{array}{ll}
\left(1 / \alpha^{2}\right)(\partial \alpha / \partial T)_{p=0}=(1 / 3)(7+4 \alpha T) & \text { (Flory) } \\
\left(1 / \alpha^{2}\right)(\partial \alpha / \partial T)_{p=0}=3+2 \alpha T & (\mathrm{vdW}) \\
\left(1 / \alpha^{2}\right)(\partial \alpha / \partial T)_{p=0}=(1 / 4)(-3 / \alpha T+6+5 \alpha T)
\end{array}
$$

(Guggenheim)

$$
\left(1 / \alpha^{2}\right)(\partial \alpha / \partial T)_{p=0}=(1 / 2)(-1 / \alpha T+4+3 \alpha T)
$$

respectively, while no corresponding explicit form can be obtained from the Frisch and Thiele equations. The curve calculated from the FHN equation comes closer to the experimental curves than do the curves from other equations. The values of $\left(1 / \alpha^{2}\right)(\partial \alpha / \partial T)_{p=0}$ calculated according to the Flory and van der Waals equations are larger than the experimental data; these equations of state give linear relations between $\left(1 / \alpha^{2}\right)(\partial \alpha / \partial T)_{p=0}$ and $\alpha T$, contrary to the experimental results. The difference between the theoretical and experimental values becomes smaller at larger values of $\alpha T$. On the other hand, the curves of $\left(1 / \alpha^{2}\right)(\partial \alpha / \partial T)_{p=0}$ calculated according to the Frisch, Thiele and Guggenheim equations appear below the observed values, but their second derivatives are negative, which is consistent with the experimental results.

\section{Temperature Dependence of $v^{*}$ and $T^{*}$}

The characteristic parameters $v^{*}, T^{*}$, and $p^{*}$ should be independent of temperature if the reduced equation of state represents exactly the behavior of real systems. Before discussing the temperature dependence of these parameters, we examine how the reduced volume $\tilde{v}$ given by eq 28 depends on $\alpha T$. For the van der Waals, Guggenheim and FHN equations, eq 28 gives

$$
\tilde{v}=1+\mathrm{d} \alpha T /(1+\alpha T)
$$

The dashed curves in Figure 2 have been calculated according to these equations of state. The solid curves were calculated using the experimental values for the specific volume $v_{\mathrm{sp}}$ and the reduction parameter $v_{\mathrm{sp}}^{*}$ per gram was chosen arbitrarily. (The absolute values of $v_{\mathrm{sp}}^{*}$ doesn't matter in the corresponding states principle.) The Guggenheim, Frisch and Thiele equations of state overestimate the dependence of $\tilde{v}$ on $\alpha T$, while the van der Waals and Flory equations underestimate it. The FHN equation reproduces the $\alpha T$ dependence of $\tilde{v}$ very closely. 


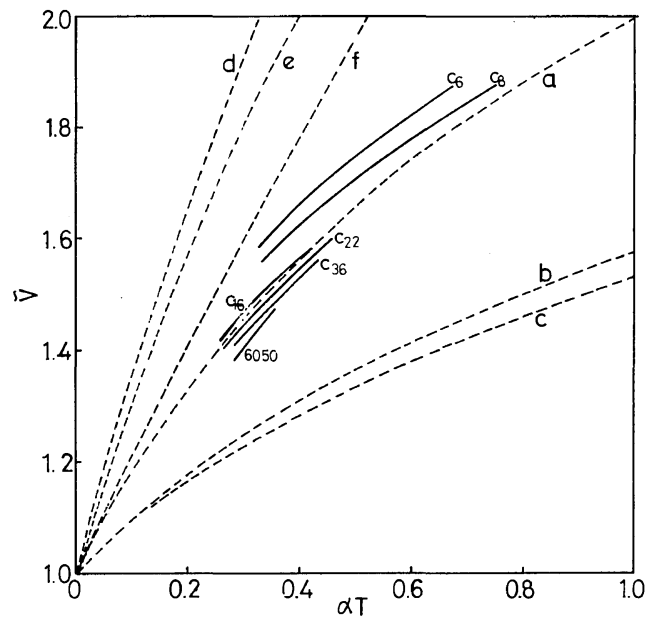

Figure 2. $\tilde{v}$ plotted against $\alpha T$ for $n$-alkanes. The solid curves are experimental. ${ }^{7}$ The dashed curves are theoretical: a, FHN equation; b, Flory equation; c, van der Waals equation; $d$, Thiele equation; e, Frisch equation; $f$, Guggenheim equation.
Although the Frisch and Thiele equations are more rigorous for the system of hard spheres than the FHN equation, the results show that these two equations, when combined with the van der Waals attractive energy, are not appropriate for $n$-alkanes.

Using the six equations of state, the average temperature dependences of $v_{\mathrm{sp}}^{*}$ and $T^{*}$ at intervals of $10^{\circ} \mathrm{C}$ are compared in Tables I and II. The FHN equation gives the least temperature dependence of either of these parameters. The Flory equation also gives good results. The results obtained from four equations of state except for the Flory and van der Waals equations show that the temperature dependence decreases with increasing the chain length of $n$-alkane molecules.

The temperature variation in $v_{\mathrm{sp}}^{*}$ and $T^{*}$ predicted by the FHN and Flory equations is also shown in Figure 3.

Table I. Temperature dependence of volume reduction parameter $v^{*}$

\begin{tabular}{lllllll}
\hline & \multicolumn{5}{c}{$\bar{c}$} \\
\cline { 2 - 6 } Alkane & FHN & Flory & vdW & Guggenheim & Thiele & Frisch \\
\cline { 2 - 6 } & -0.2 & +0.2 & +0.4 & -0.9 & -1.0 & -1.0 \\
$\mathrm{C}_{6}$ & -0.2 & +0.2 & +0.4 & -0.9 & -1.0 & -0.9 \\
$\mathrm{C}_{8}$ & -0.1 & +0.2 & +0.3 & -0.6 & -0.6 & -0.6 \\
$\mathrm{C}_{16}$ & $-0.0_{4}$ & +0.2 & +0.3 & -0.5 & -0.5 & -0.5 \\
$\mathrm{C}_{22}$ & $-0.0_{3}$ & +0.2 & +0.3 & -0.4 & -0.5 & -0.5 \\
$\mathrm{C}_{36}$ & $+0.0_{1}$ & +0.2 & +0.3 & -0.3 & -0.3 & -0.4 \\
Marlex 50 & +0.1 & +0.3 & +0.3 & -0.3 & -0.3 & -0.3 \\
Marlex 6050 & & & & & & \\
\end{tabular}

a The results are shown as average values of relative deviation at intervals of $10^{\circ} \mathrm{C}$.

Table II. Temperature dependence of temperature reduction parameter $T^{*}$

\begin{tabular}{lcccccc}
\hline & \multicolumn{5}{c}{$\left(\bar{c}\left(T_{t+10}^{*}-T_{t}^{*}\right) / T_{t}{ }^{*} \times 10^{2 \mathrm{a}}\right.$} \\
\cline { 2 - 7 } Alkane & FHN & Flory & $\mathrm{vdW}$ & Guggenheim & Frisch & Thiele \\
\hline $\mathrm{C}_{6}$ & -0.4 & +0.6 & +1.1 & -1.8 & -2.0 & -2.0 \\
$\mathrm{C}_{8}$ & -0.4 & +0.4 & +0.8 & -1.7 & -1.8 & -1.9 \\
$\mathrm{C}_{16}$ & -0.2 & +0.8 & +1.1 & -1.6 & -1.7 & -1.6 \\
$\mathrm{C}_{22}$ & -0.1 & +0.7 & +1.0 & -1.4 & -1.5 & -1.4 \\
$\mathrm{C}_{36}$ & -0.1 & +0.7 & +1.0 & -1.2 & -1.3 & -1.2 \\
Marlex 50 & $+0.0_{4}$ & +0.8 & +1.0 & -1.1 & -1.2 & -0.7 \\
Marlex 6050 & +0.2 & +0.9 & +1.1 & -0.8 & -0.9 & -0.5 \\
\hline
\end{tabular}

a The results are shown as average values of relative deviation at intervals of $10^{\circ} \mathrm{C}$. 

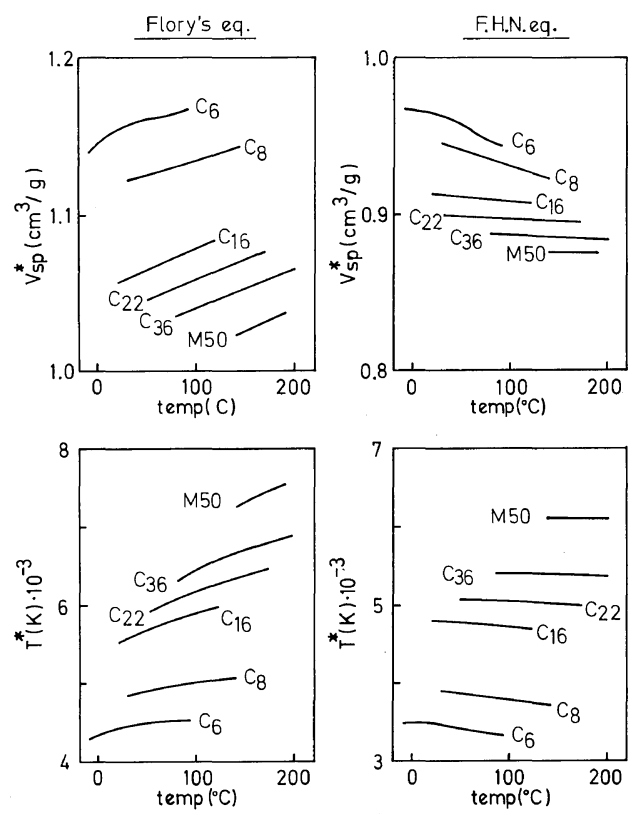

Figure 3. Temperature dependencies of $v_{\mathrm{sp}}^{*}$ and $T^{*}$ calculated from the FHN and Flory equations.

\section{Thermal Pressure Coefficients and Temperature Dependence of $p^{*}$}

The internal pressure, i.e., the thermal pressure coefficient $\gamma$ multiplied by temperature $T$, is directly related to the shape of the configurational energyvolume curve by,

$$
\gamma T=\left(\frac{\partial E}{\partial V}\right)_{T}=\frac{E^{*}}{V^{*}}\left(\frac{\partial \tilde{E}}{\partial \widetilde{V}}\right)_{\tilde{T}}=p^{*}\left(\frac{\partial \tilde{E}}{\partial \tilde{V}}\right)_{\tilde{T}}
$$

Consider the curves of $\gamma T$ against $\alpha T$ for alkanes of different chain length. If the corresponding states principle holds, these curves should fall on a single master curve when shifted by $p_{\mathrm{R}}{ }^{*} / p^{*}$ where $p_{\mathrm{R}}{ }^{*}$ is the reduction pressure parameter for $n$-alkane reference. In Figure 4, the experimental values (shown by circles) of Flory et al. ${ }^{7}$ for $\mathrm{C}_{6}, \mathrm{C}_{8}, \mathrm{C}_{16}, \mathrm{C}_{22}, \mathrm{C}_{36}$, and Marlex 50 are plotted against $\alpha T$. In this case, no shifting was necessary to obtain a single curve within experimental error. This suggests that the corresponding states principle nearly holds for $n$ alkanes and that the end group (the methyl group) and the internal group (the methylene group) may be quite similar with respect to the intermolecular contact energy, as was demonstrated by Patterson. ${ }^{17}$

Each equation of state gives $\gamma T$ as a function of

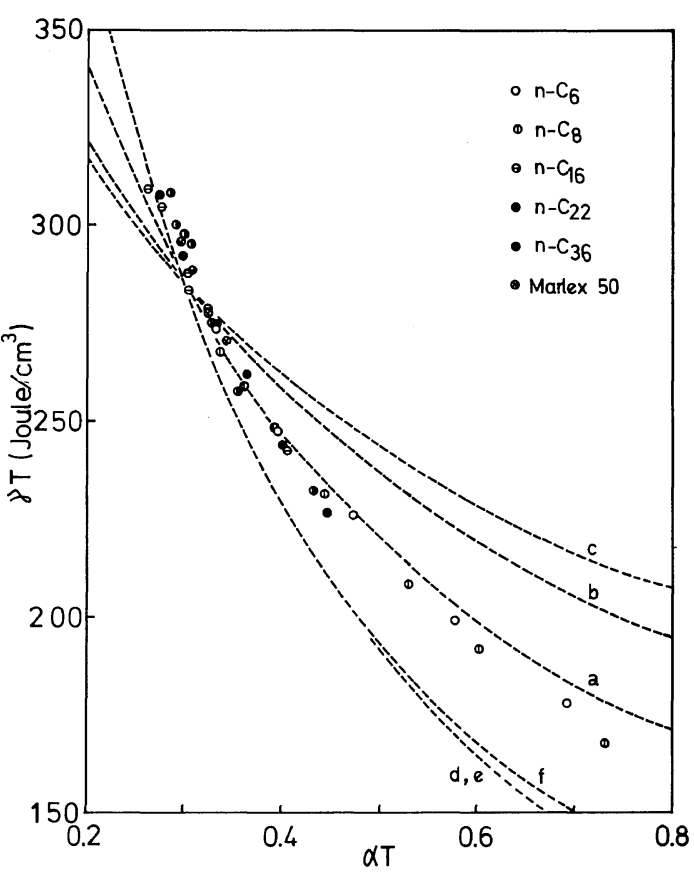

Figure 4. $\gamma T$ plotted against $\alpha T$ for $n$-alkanes. The points are experimental. ${ }^{7}$ The dashed curves are theoretical: a, FHN equation; b, Flory equation; c, van der Waals equation; $d$, Thiele equation; $e$, Frisch equation; $f$, Guggenheim equation.

$\alpha T$ if $p^{*}$ is known, as shown in eq 29 , i.e.,

$$
\gamma T=p^{*} \tilde{v}^{-2}
$$

where $\tilde{v}$ is a function of $\alpha T$ as shown in eq 28. In Figure 4 , the values of $\gamma T$ calculated from eq 37 according to each equation are represented by a broken curve. In calculating these $\gamma T-\alpha T$ curves, $p^{*}$ was taken to be independent of temperature and chosen so as to give $\gamma T=285 \mathrm{Jcm}^{-3}$ at $\alpha T=0.3$. The FHN equation gives a good representation of the experimental results.

If $p^{*}$ is calculated from the experimental values of $\gamma T$ and $\alpha T$ by eq 37 or 29 , the value of $p^{*}$ increases by $5 \%$ in the FHN equation and decreases by 20 and $25 \%$ in the Flory and van der Waals equations, respectively, and increases by 15,20 , and $20 \%$ in the Guggenheim, Thiele and Frisch equations, respectively, over an $\alpha T$ range from 0.25 to 0.7 .

\section{Consideration of Volume Dependence of Con- figurational Energy}

In the preceding sections, some equations of state 
with the van der Waals attractive energy were treated. It is interesting to combine an equation of state for hard spheres with the Lennard-Jones type potential. Simha and Havlik ${ }^{18}$ showed that the equation of state derived from the cell theory of Prigogine et al. is valid in the range of a comparatively small reduced volume $\tilde{v}$, and that the corresponding states principle holds throughout the whole range of $\tilde{v}$.

In this section, we examine the Thiele equation, which is more rigorous for hard sphere systems, combined with the Lennard-Jones type potential. Using the intermolecular potential of the LennardJones $(m, n)$ type, we can express the reduced configurational energy as,

$$
\begin{aligned}
\tilde{E} & =-\frac{1}{n-m}\left(n \tilde{v}^{-m / 3}-m \tilde{v}^{-n / 3}\right) & & (\tilde{v} \geqq 1) \\
& =+\infty & & (\tilde{v}<1)
\end{aligned}
$$

or

$$
\begin{aligned}
\tilde{E} & =-\frac{1}{n-m}\left(n \tilde{v}^{-m / 3}-m \tilde{v}^{-n / 3}\right) & & \left(\tilde{v} \geqq(n / m)^{3 /(m-n)}\right) \\
& =+\infty & & \left(\tilde{v}<(n / m)^{3 /(m-n)}\right)
\end{aligned}
$$

In eq 39 , the reduction parameter $v^{*}$ is expressed by,

$$
v^{*}=\frac{4}{3} \pi\left(\frac{R^{*}}{2}\right)^{3}
$$

where $R^{*}$ is the distance at which the LennardJones $(m, n)$ potential becomes minimum. On the other hand, in eq $38, v^{*}$ is given by $(4 / 3) \pi(\sigma / 2)^{3}$ as in the previous sections. For the case of $(m, \infty), R^{*}$ coincides with $\sigma$ and the results calculated from the two potentials described above with each other. The schematic representations of eq 38 and 39 for certain combinations of $m$ and $n$ are shown in Figure 5.

The reduced equation of state corresponding to eq 14 is given by,

$$
\frac{\tilde{p} \tilde{v}}{\tilde{T}}=H(v)-\frac{m n}{3(n-m) \tilde{T}}\left(\tilde{v}^{-m / 3}-\tilde{v}^{-n / 3}\right)
$$

and at zero pressure,

$$
\tilde{T}=\frac{m n}{3(n-m) H(\tilde{v})}\left(\tilde{v}^{-m / 3}-\tilde{v}^{-n / 3}\right)
$$

The term $H(\tilde{v})$ of the Thiele equation combined with eq 38 is expressed by eq 18 , but when it is combined with eq $39, H(\tilde{v})$ should be modified to,

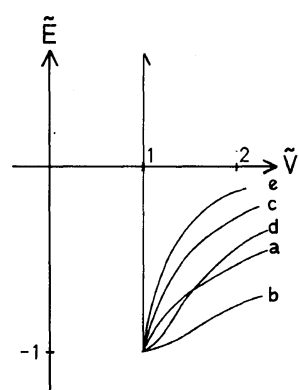

(A)

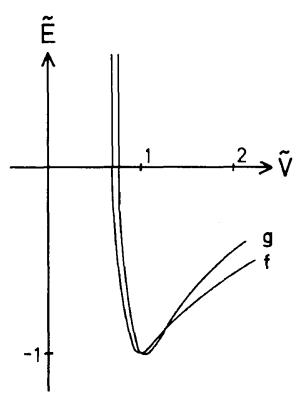

(B)
Figure 5. Schematic diagrams of reduced configurational energy $\tilde{E}$ plotted against reduced volume $\tilde{v}$. (A): The equation 38 with the combination of the following $(m, n): \mathrm{a},(3, \infty)$; b, $(3,12)$; c, $(6, \infty)$; d, $(6,12)$; e, $(9, \infty)$. (B): The equation 39 with the following combination of $(m, n): \mathrm{f},(3,27) ; \mathrm{g},(6,12)$.

$$
H(\tilde{v})=\left(\tilde{v}^{2}+2 B \tilde{v}+3 B^{2}\right) /(\tilde{v}-B)^{2}
$$

where $B$ is given by,

$$
B=\left(\frac{m}{n}\right)^{3 /(n-m)}=\left(\frac{\sigma}{R^{*}}\right)^{3}
$$

From eq 42 , the dependence of $\alpha T$ on $\tilde{v}$ at zero pressure is,

$$
(\alpha T)^{-1}=-\tilde{v}[\ln H(\tilde{v})]^{\prime}-J(\tilde{v})
$$

where $J(\tilde{v})$, the term related to the configurational energy, is expressed by,

$$
J(\tilde{v})=\left[(m / 3) \tilde{v}^{-m / 3}-(n / 3) \tilde{v}^{-n / 3}\right] /\left(\tilde{v}^{-m / 3}-\tilde{v}^{-n / 3}\right)
$$

The relation of $\left(1 / \alpha^{2}\right)(\partial \alpha / \partial T)_{p=0}$ to $\tilde{v}$ is given by,

$$
\begin{aligned}
& \left(1 / \alpha^{2}\right)(\partial \alpha / \partial T)_{p=0} \\
& =\frac{\tilde{v}^{2}[\ln H(\tilde{v})]^{\prime \prime}+\tilde{v}[\ln H(\tilde{v})]^{\prime}+\tilde{v}[J(\tilde{v})]^{\prime}}{-\tilde{v} \ln H(\tilde{v})-J(\tilde{v})} \\
& \quad+\tilde{v}[\ln H(\tilde{v})]^{\prime}+J(\tilde{v})
\end{aligned}
$$

The quantity $\gamma T$ is represented by,

$$
\gamma T=\frac{1}{3} \frac{m n}{n-m} p^{*} \tilde{v}^{-1}\left(\tilde{v}^{-m / 3}-\tilde{v}^{-n / 3}\right)
$$

Figures 6 and 7 show the relationship between $\left(1 / \alpha^{2}\right)(\partial \alpha / \partial T)_{p=0}$ and $\alpha T$ and that between $\gamma T$ and $\alpha T$, respectively. In these figures, the dashed curves (a, b, c, d, e) were calculated by eq 18 and 38 . The $(6,12)$ choice among five $(m, n)$ gives good results for both $\left(1 / \alpha^{2}\right)(\partial \alpha / \partial T)-\alpha T$ and $\gamma T-\alpha T$. Although 
the $(6, \infty)$ choice gives a good representation of the relation between $\left(1 / \alpha^{2}\right)(\partial \alpha / \partial T)$ and $\alpha T$, it gives $\gamma T$ which decreases rapidly with an increase in $\alpha T$.

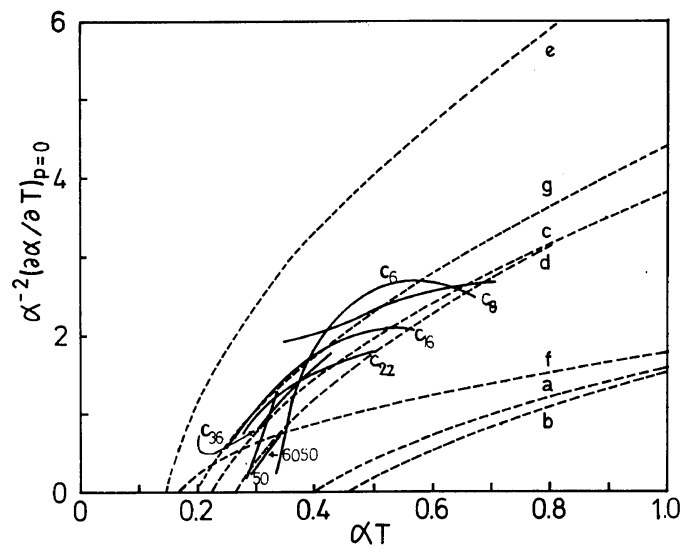

Figure 6. $\left(1 / \alpha^{2}\right)(\partial \alpha / \partial T)_{p=0}$ plotted against $\alpha T$ for $n$ alkanes. The full curves are experimental. ${ }^{7}$ The dashed curves $(a, b, c, d, e)$ are calculated by combination of the Thiele equation and eq 38 with the following $(m, n)$ : a, $(3, \infty) ; \mathrm{b},(3,6) ; \mathrm{c},(6, \infty) ; \mathrm{d},(6,12) ; \mathrm{e},(9, \infty)$. The dashed curves ( $f, g)$ are calculated by combination of the Thiele equation and eq 39 with the following $(m, n): \mathrm{f}$, $(3,27)$; $g,(6,12)$.

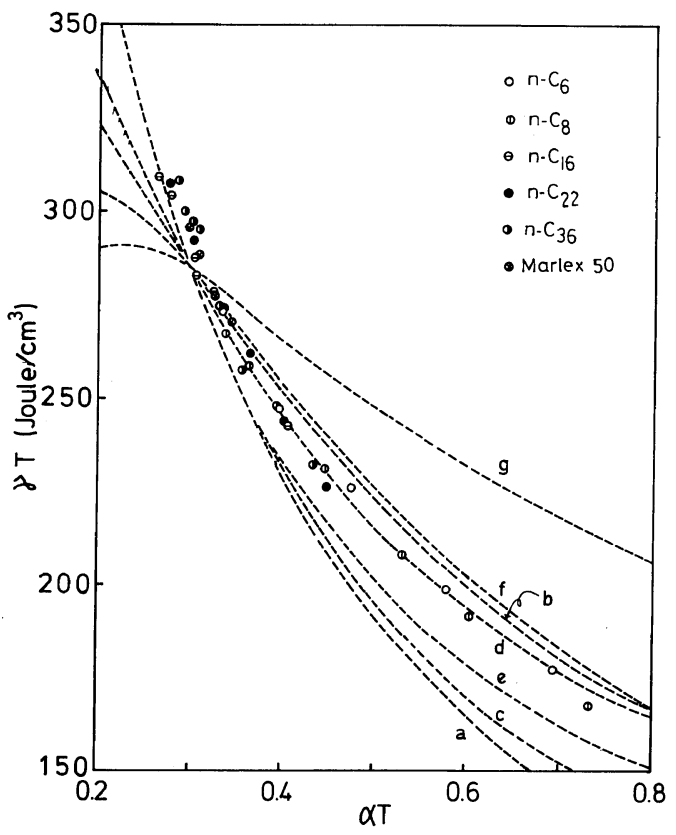

Figure 7. $\gamma T$ plotted against $\alpha T$ for $n$-alkanes. The points are experimental. ${ }^{7}$ The dashed curves are theoretical; the meaning for $a-f$ is the same as that in Figure 6 .
The calculation according to eq 39 and 43 was carried out for two cases: $f,(3,27)$ and $g,(6,12)$. The $(3,27)$ choice leads to the configurational energy suggested by Hildebrand ${ }^{19,20}$ to account for the values of $(\partial E / \partial V)_{T}$ at high density| for low molecular weight substances. As shown in Figure 7, the relation $\gamma T-\alpha T$ is fairly well expressed by the $(3,27)$ choice. But this choice cannot reproduce the observed $\left(1 / \alpha^{2}\right)(\partial \alpha / \partial T)$, as shown in Figure 6 . The choice $(6,12)$ is not good for representing the $\gamma T-\alpha T$ relation, contrary to the results obtained from eq 18 and 38.

These results show that the Thiele equation gives a good representation of the experimental data for $n$-alkanes when combined not with the van der Waals energy inversely proportional to volume but with the Lennard-Jones $(6,12)$ potential given by eq 39. The results also show that the parameter $p^{*}$ calculated according to this equation is almost independent of either chain length or temperature within experimental error.

\section{APPLICATION TO OTHER SMALL MOLECULES AND POLYMERS}

As shown in the previous sections, when the hardsphere repulsive part is combined with the van der Waals attractive part, the FHN and Flory equations are more suitable for $n$-alkanes than are the other four equations. In this section, we examine the applicability of these two equations to other pure liquids including polymer liquids.

It is seen in Figure 8 that the observed relations between $\left(1 / \alpha^{2}\right)(\partial \alpha / \partial T)_{p=0}$ and $\alpha T$ approximately

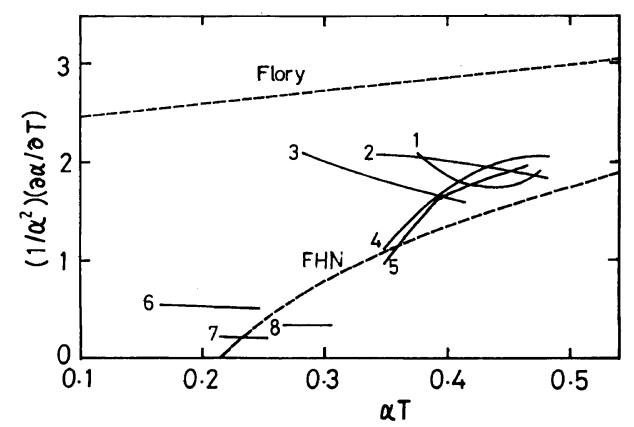

Figure 8. The full curves are experimental: 1 , methyl ethyl ketone ${ }^{5} ; 2, \mathrm{C}_{6} \mathrm{H}_{12}{ }^{20} ; 3, \mathrm{C}_{6} \mathrm{H}_{5} \mathrm{Cl}^{6} ; 4, \mathrm{CCl}_{4}{ }^{20} ; 5$, $\mathrm{C}_{6} \mathrm{H}_{6}{ }^{22} ; 6, \mathrm{PIB}^{3} ; 7, \mathrm{PS}^{5} ; 8, \mathrm{PDMS}^{6}$. The dashed curves theoretical. 
Table III. Comparison of $v_{\mathrm{sp}}, \alpha$, and $\gamma$ calculated from the Flory and FHN equations with the experimental values

\begin{tabular}{|c|c|c|c|c|c|c|c|c|c|c|}
\hline \multirow{3}{*}{ Liquid } & \multirow{3}{*}{$\mathrm{Temp} /{ }^{\circ} \mathrm{C}$} & \multicolumn{3}{|c|}{$v_{\mathrm{sp}} / \mathrm{cm}^{3} \mathrm{~g}^{-1}$} & \multicolumn{3}{|c|}{$\alpha \times 10^{3} / \mathrm{deg}^{-1}$} & \multicolumn{3}{|c|}{$\gamma /$ bar deg $^{-1}$} \\
\hline & & \multirow{2}{*}{ Exptl } & \multicolumn{2}{|c|}{ Calcd } & \multirow{2}{*}{ Exptl } & \multicolumn{2}{|c|}{ Calcd } & \multirow{2}{*}{ Expt1 } & \multicolumn{2}{|c|}{ Calcd } \\
\hline & & & FHN & Flory & & FHN & Flory & & FHN & Flory \\
\hline $\mathrm{C}_{6} \mathrm{H}_{12}$ & $\begin{array}{l}25 \\
70\end{array}$ & $\begin{array}{l}1.2922^{\mathrm{a}} \\
1.3679^{\mathrm{a}}\end{array}$ & $\begin{array}{l}1.2920 \\
1.3688\end{array}$ & $\begin{array}{l}1.2923 \\
1.3704\end{array}$ & $\begin{array}{l}1.217^{\mathrm{a}} \\
1.365^{\mathrm{a}}\end{array}$ & $\begin{array}{l}1.235 \\
1.339\end{array}$ & $\begin{array}{l}1.200 \\
1.422\end{array}$ & $\begin{array}{r}10.67^{\mathrm{b}} \\
8.08^{\mathrm{b}}\end{array}$ & $\begin{array}{r}10.66 \\
8.25\end{array}$ & $\begin{array}{r}10.66 \\
8.23\end{array}$ \\
\hline $\mathrm{C}_{6} \mathrm{H}_{6}$ & $\begin{array}{l}25 \\
70\end{array}$ & $\begin{array}{l}1.1444^{\mathrm{c}} \\
1.2122^{\mathrm{c}}\end{array}$ & $\begin{array}{l}1.1444 \\
1.2119\end{array}$ & $\begin{array}{l}1.1447 \\
1.2133\end{array}$ & $\begin{array}{l}1.223^{\mathrm{c}} \\
1.346^{\mathrm{c}}\end{array}$ & $\begin{array}{l}1.227 \\
1.328\end{array}$ & $\begin{array}{l}1.192 \\
1.411\end{array}$ & $\begin{array}{r}12.64^{\mathrm{b}} \\
9.54^{\mathrm{b}}\end{array}$ & $\begin{array}{r}12.55 \\
9.70\end{array}$ & $\begin{array}{r}12.51 \\
9.67\end{array}$ \\
\hline $\mathrm{C}_{6} \mathrm{H}_{5} \mathrm{Cl}$ & $\begin{array}{l}25 \\
70\end{array}$ & $\begin{array}{l}0.9091^{\mathrm{d}} \\
0.9514^{\mathrm{d}}\end{array}$ & $\begin{array}{l}0.9090 \\
0.9510\end{array}$ & $\begin{array}{l}0.9092 \\
0.9518\end{array}$ & $\begin{array}{l}0.967^{\mathrm{d}} \\
1.054^{\mathrm{d}}\end{array}$ & $\begin{array}{l}0.984 \\
1.026\end{array}$ & $\begin{array}{l}0.957 \\
1.087\end{array}$ & $\begin{array}{l}13.08^{\mathrm{d}} \\
10.35^{\mathrm{d}}\end{array}$ & $\begin{array}{l}13.11 \\
10.41\end{array}$ & $\begin{array}{l}13.11 \\
10.39\end{array}$ \\
\hline MEK & $\begin{array}{l}25 \\
70\end{array}$ & $\begin{array}{l}1.2502^{\mathrm{e}} \\
1.3309^{\mathrm{e}}\end{array}$ & $\begin{array}{l}1.2502 \\
1.3303\end{array}$ & $\begin{array}{l}1.2505 \\
1.3320\end{array}$ & $\begin{array}{l}1.308^{\mathrm{e}} \\
1.467^{\mathrm{e}}\end{array}$ & $\begin{array}{l}1.319 \\
1.451\end{array}$ & $\begin{array}{l}1.281 \\
1.542\end{array}$ & $\begin{array}{r}11.42^{\mathrm{e}} \\
9.91^{\mathrm{e}}\end{array}$ & $\begin{array}{r}11.43 \\
8.77\end{array}$ & $\begin{array}{r}11.42 \\
8.75\end{array}$ \\
\hline $\mathrm{CCl}_{4}$ & $\begin{array}{l}25 \\
70\end{array}$ & $\begin{array}{l}0.6311^{\mathrm{a}} \\
0.6688^{\mathrm{a}}\end{array}$ & $\begin{array}{l}0.6311 \\
0.6686\end{array}$ & $\begin{array}{l}0.6312 \\
0.6694\end{array}$ & $\begin{array}{l}1.229^{\mathrm{a}} \\
1.363^{\mathrm{a}}\end{array}$ & $\begin{array}{l}1.236 \\
1.340\end{array}$ & $\begin{array}{l}1.201 \\
1.423\end{array}$ & $\begin{array}{r}11.42^{\mathrm{b}} \\
8.58^{\mathrm{b}}\end{array}$ & $\begin{array}{r}11.32 \\
8.77\end{array}$ & $\begin{array}{r}11.32 \\
8.74\end{array}$ \\
\hline PDMS & $\begin{array}{r}50 \\
100\end{array}$ & $\begin{array}{l}1.0549^{\mathrm{d}} \\
1.1046^{\mathrm{d}}\end{array}$ & $\begin{array}{l}1.0551 \\
1.1048\end{array}$ & $\begin{array}{l}1.0558 \\
1.1051\end{array}$ & $\begin{array}{l}0.914^{\mathrm{d}} \\
0.929^{\mathrm{d}}\end{array}$ & $\begin{array}{l}0.902 \\
0.940\end{array}$ & $\begin{array}{l}0.858 \\
0.973\end{array}$ & $\begin{array}{l}6.57^{\mathrm{d}} \\
4.90^{\mathrm{d}}\end{array}$ & $\begin{array}{l}6.36 \\
5.02\end{array}$ & $\begin{array}{l}6.35 \\
5.02\end{array}$ \\
\hline PIB & $\begin{array}{r}50 \\
150\end{array}$ & $\begin{array}{l}1.1059^{\mathrm{f}} \\
1.1706^{\mathrm{f}}\end{array}$ & $\begin{array}{l}1.1056 \\
1.1704\end{array}$ & $\begin{array}{l}1.1068 \\
1.1716\end{array}$ & $\begin{array}{l}0.560^{\mathrm{f}} \\
0.577^{\mathrm{f}}\end{array}$ & $\begin{array}{l}0.572 \\
0.570\end{array}$ & $\begin{array}{l}0.529 \\
0.614\end{array}$ & $\begin{array}{r}10.19^{\mathrm{f}} \\
6.78^{\mathrm{f}}\end{array}$ & $\begin{array}{r}10.06 \\
6.86\end{array}$ & $\begin{array}{r}10.04 \\
6.84\end{array}$ \\
\hline
\end{tabular}

${ }^{\mathrm{a}}$ Reference $21 .{ }^{\mathrm{b}}$ Reference $22 .{ }^{\mathrm{c}}$ Reference $23 .{ }^{\mathrm{d}}$ Reference $6 .{ }^{\mathrm{e}}$ Reference $5 .{ }^{\mathrm{f}}$ Reference 3.

fall on a single curve, though the tendency of curves for polymers is somewhat different from that for small molecules. The curve calculated from the FHN equation agrees better with the experimental values than that calculated from the Flory equation.

In Table III, the values of $v_{\mathrm{sp}}, \alpha$ and $\gamma$ calculated from eq 21 and $27-29$ are compared with the experimental values. $^{5,6,21-23}$ These calculations were carried out using the characteristic parameters $v_{\mathrm{sp}}^{*}, p^{*}$ and $T^{*}$ selected at $40^{\circ} \mathrm{C}$ for the small molecules, and at 80,150, and $100^{\circ} \mathrm{C}$ for poly(dimethylsiloxane) (PDMS), polystyrene (PS), and polyisobutylene (PIB), respectively. The values calculated for the small molecules from the FHN equation are as a whole in better agreement with the experimental values than those from the Flory equation. Especially, the FHN equation is satisfactory for $\alpha$ of the liquids examined except for chlorobenzene and cyclohexane. For these two liquids, the values of $\alpha$ calculated by the FHN equation deviate from the observed values as much as those calculated by the Flory equation; the experimental values of $\left(1 / \alpha^{2}\right)(\partial \alpha / \partial T)_{p=0}$ appear between the values calculated from the two equations, as seen in
Figure 8. It is also noted that the FHN equation can reproduce the experimental values of $v_{\mathrm{sp}}$ and $\alpha$ for the polymers better than the Flory equation can.

The values of $\gamma$ calculated from the FHN equation are nearly the same as those obtained from the Flory equation. This arises from the fact that the same type of attractive energy, i.e., the van der Waals type, is used in both equations.

Figure 9 shows the pressure dependence of the volume of the polymers. Calculations were made using the characteristic parameters obtained from the data at atmospheric pressure and at the respective temperatures. The results suggest that the FHN equation is better than the Flory equation for predicting the densities of the polymers at high pressure.

\section{DISCUSSION}

Hildebrand ${ }^{18,19}$ and Frank ${ }^{25}$ maintain that the configurational energy $E$ is expressed by $E=-$ const. $/ V^{m}$, where $m$ is nearly equal to unity. Gee et $a l .{ }^{26}$ have estimated $m$ from internal pressure data $P_{\mathrm{i}}$ and $\Delta H_{\mathrm{v}}$ and obtained $m$ values for $C_{5}, \mathrm{C}_{6}$, 


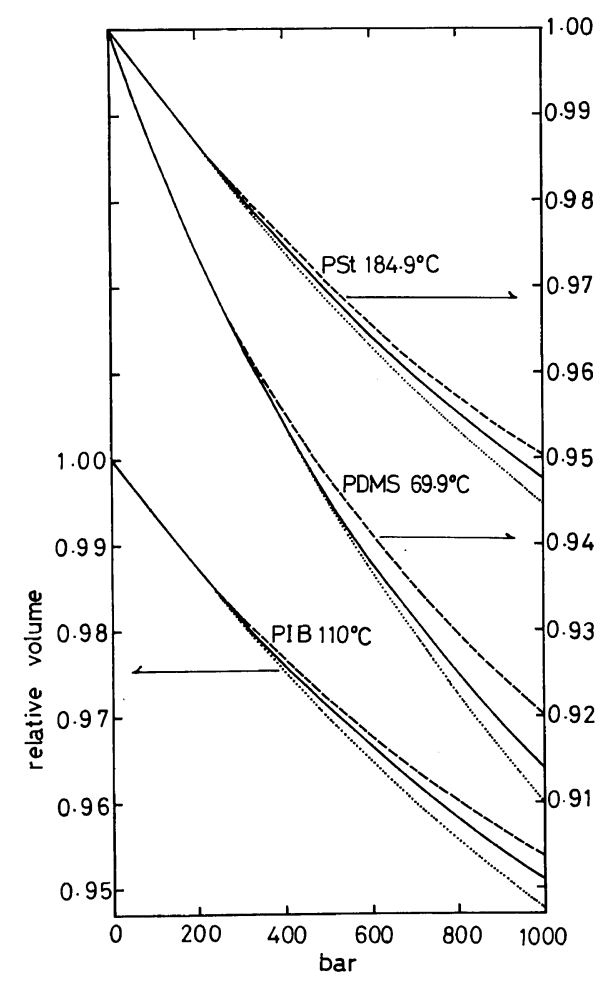

Figure 9. The full and dashed curves were calculated from the FHN and Flory equations, respectively. The dotted curves are experimental. ${ }^{24}$

$\mathrm{C}_{7}, \mathrm{C}_{8}, \mathrm{C}_{9}, \mathrm{C}_{10}$ and polyethylene as $1.09,1.06,1.09$, $1.10,1.11,1.11$ and 1.11 , respectively. These results show that the configurational energy for $n$-alkanes can be expressed fairly well by van der Waals inverse volume dependence. The Thiele and Frisch equations are excellent for application to hard spheres, but when combined with the van der Waals energy, these do not give a good representation of experimental data. On the contrary, though it is an empirical one and has no theoretical meaning, but when combined with the van der Waals energy, the FHN equation gives a good representation of experimental data for $n$-alkanes. The fact that satisfactory results were obtained by the FHN equation may be due to the deviation of $n$-alkanes from a hard sphere model. We again note that the equation of state by Flory gives an equally good representation of experimental data for $n$-alkanes.

The Thiele equation combined with the LennardJones $(6,12)$ potential in the form of eq 38 gives a good representation for experimental data, but the
Lennard-Jones $(6,12)$ potential expressed by eq 39 is not suitable for the perturbation energy for the $n$ alkane system. Patterson et al. ${ }^{17}$ discussed $\left(1 / \alpha^{2}\right)(\partial \alpha / \partial T)_{p=0}$ as a function of $\alpha T$, by combining Prigogine's smoothed potential cell model for chain molecular liquids with the potential energy of the $(m, n)$ type. The combination with the $(6,12)$ potential yielded the general shape of the experimental curves, but the values of $\left(1 / \alpha^{2}\right)(\partial \alpha / \partial T)_{p=0}$ were too large for any values of $\alpha T$. They also showed that the combination with the $(6,12)$ potential gave a larger temperature dependence of $P^{*}$ than that calculated by Flory's theory. We thus conclude that the combination of a suitable equation of state for the hard sphere model with a suitable configurational energy is essential for accurately predicting thermodynamic properties of liquids.

\section{REFERENCES}

1. P. J. Flory, R. A. Orwoll, and A. Vrij, J. Am. Chem. Soc., 86, 3507 (1964).

2. P. J. Flory, J. Am. Chem. Soc., 87, 1833 (1965); A. Abe and P. J. Flory, ibid., 87, 1838 (1965).

3. P. J. Flory, J. E. Ellenson, and B. E. Eichinger, Macromolecules, 1, 279 (1968); B. E. Eichinger and P. J. Flory, ibid., 1, 285 (1968); P. J. Flory, B. E. Eichinger, and R. A. Orwoll, ibid., 1, 287 (1968).

4. B. E. Eichinger and P. J. Flory, Trans. Faraday Soc., 64, 2035, 2053, 2061, 2066 (1968).

5. H. Höcker, G. J. Blake, and P. J. Flory, Trans. Faraday Soc., 67, 2251 (1971); P. J. Flory and H. Höcker, ibid., 67, 2258 (1971); H. Höcker and P. J. Flory, ibid., 67, 2270 (1971); H. Höcker, H. Shih, and P. J. Flory, ibid., 67, 2275 (1971).

6. H. Shih and P. J. Flory, Macromolecules, 5, 758 (1972); P. J. Flory and H. Shih, ibid., 5, 761 (1972).

7. R. A. Orwoll and P. J. Flory, J. Am. Chem. Soc., 89, 6814 (1967).

8. H. C. Longuet-Higgins and B. Widom, Mol. Phys., 8, 549 (1964).

9. D. Henderson, Ed., "Physical Chemistry," Vol. VIIIA, Liquid State, Academic Press, New York and London, 1971; S. A. Rice and P. Gray, "The Statistical Mechanics of Simple Liquids," Interscience Publishers, Inc., New York, 1965.

10. E. A. Guggenheim, Mol. Phys., 9, 199 (1965).

11. H. Reiss, H. L. Frisch, and J. L. Lebowitz, J. Chem. Phys., 31, 369 (1959); E. H. Helfand and H. L. Frisch, J. Chem. Phys., 34, 1037 (1961).

12. E. Thiele, J. Chem. Phys., 39, 474 (1963).

13. J. S. Rowlinson, Discuss. Faraday Soc., 49, 30 (1970).

14. M. Kac, Phys. Fluids, 2, 8 (1959); M. Kac, G. E. Uhlenbeck, and P. C. Hemmer, J. Math. Phys., 4, 
216, 229 (1963).

15. N. S. Snider and T. M. Herrington, J. Chem. Phys., 47, 2248 (1967).

16. R. L. Scott and P. H. van Konynenburg, Discuss. Faraday Soc., 49, 87 (1970).

17. D. Patterson and J. M. Bardin, Trans. Faraday Soc., 66, 321 (1970).

18. R. Simha and A. J. Havlik, J. Am. Chem. Soc., 86, 197 (1964).

19. J. H. Hildebrand and R. L. Scott, "The Solubility of Nonelectrolytes," 3rd ed, Reinhold Publishing Corporation, New York, N.Y., 1950.

20. J. H. Hildebrand and R. L. Scott, "Regular
Solutions," Prentice-Hall Inc., Englewood Cliffs, N.J., 1962.

21. S. E. Wood and J. A. Gray, J. Am. Chem. Soc., 74, 3729 (1952).

22. G. A. Holder and E. Whalley, Trans. Faraday Soc., 58, 2095 (1962).

23. S. E. Wood and J. P. Brusie, J. Am. Chem. Soc., 65, 1891 (1943).

24. S. Beret and J. M. Prausnitz, Macromolecules, 8, 536 (1975).

25. H. S. Frank, J. Chem. Phys., 13, 478, 493 (1945).

26. G. Allen, G. Gee, and J. G. Wilson, Polymer, 1, 456 (1960). 